\section{Neuronal SIRT1 regulates endocrine and behavioral responses to calorie restriction}

\author{
Dena E. Cohen, Andrea M. Supinski, \\ Michael S. Bonkowski, Gizem Donmez, \\ and Leonard P. Guarente ${ }^{1}$
}

Paul F. Glenn Laboratory, Department of Biology, Massachusetts Institute of Technology, Cambridge, Massachusetts 02139, USA

Mammalian life span can be extended by both calorie restriction $(C R)$ and mutations that diminish somatotropic signaling. Sirt1 is a mediator of many effects of CR in mammals, but any role in controlling somatotropic signaling has not been shown. Since the somatotropic axis is controlled by the brain, we created mice lacking Sirt1 specifically in the brain and examined the impacts of this manipulation on somatotropic signaling and the CR response. These mutant mice displayed defects in somatotropic signaling when fed ad libitum, and defects in the endocrine and behavioral responses to $C R$. We conclude that Sirt1 in the brain is a link between somatotropic signaling and $C R$ in mammals.

Supplemental material is available at http://www.genesdev.org.

Received July 2, 2009; revised version accepted October 20, 2009.

Mammalian life span can be extended by both dietary and genetic interventions. Calorie restriction (CR), a reduction of food intake while maintaining adequate nutrition, dramatically extends the life span in both invertebrates and mammals (for review, see Bordone and Guarente 2005). In multiple species, the beneficial effects of CR have been linked to the function of the sirtuin family of $\mathrm{NAD}^{+}$-dependent protein deacetylases (Sirt1 in mouse). In yeast, fruit flies, and possibly mice, genetic ablation of Sirtl orthologs blocks the extension of life span by CR (Lin et al. 2000; Rogina and Helfand 2004; Li et al. 2008). Because sirtuins require $\mathrm{NAD}^{+}$as an essential cofactor for their deacetylase activity, they are thought to read the metabolic state of an organism and direct changes in transcription and metabolism in response (Imai et al. 2000). Sirt1 regulates multiple metabolic pathways in mammals, and is thought to play a critical role in directing protective changes in response to alterations in nutritional status (Guarente 2009).

Genetic and physiological analysis of Sirt1 in mice supports the model that this sirtuin mediates the beneficial effects of CR. Sirt1 protein levels increase in many, but not all, mammalian tissues during CR (Cohen et al. 2004; Chen et al. 2008). Furthermore, whole-body Sirt1

[Keywords: Sirt1; growth hormone; calorie restriction; aging] ${ }^{1}$ Corresponding author.

E-MAIL leng@mit.edu; FAX (617) 253-8699.

Article is online at http://www.genesdev.org/cgi/doi/10.1101/gad.1839209. knockout mice show neither the increase in physical activity nor many of the physiological changes normally associated with CR (Chen et al. 2005; Boily et al. 2008; Li et al. 2008). Conversely, transgenic mice that overexpress Sirtl show metabolic phenotypes that partially overlap those of CR (Bordone et al. 2007; Banks et al. 2008; Pfluger et al. 2008). Finally, small molecules that increase the enzymatic activity of Sirtl induce transcriptional changes that significantly overlap changes induced by CR (Barger et al. 2008a,b; Pearson et al. 2008).

In addition to $\mathrm{CR}$, mouse life span can be extended by single-gene mutations. Collectively, these mutations point to the importance of the somatotropic axis as a major regulator of mouse life span. Long-lived Ames and Snell dwarf mice harbor mutations that disrupt pituitary development, thereby reducing levels of growth hormone (GH) as well as several other pituitary hormones (for review, see Tatar et al. 2003). These mutations also diminish levels of insulin-like growth factor 1 (IGF-1), a downstream mediator of many of the effects of GH whose role in the regulation of life span is less certain (Holzenberger et al. 2003; Ladiges et al. 2009). It has long been known that CR down-regulates somatotropic signaling in mice, as indicated by a reduction in circulating IGF1 (Al-Regaiey et al. 2005), suggesting that CR and mutations that block somatotropic signaling may extend life span in part through shared mechanisms. Indeed, mice in which GH signaling is blocked by ablation of the GH receptor receive no further extension of overall longevity when placed on a CR diet (Bonkowski et al. 2006). The mechanisms by which CR might direct changes in the somatotropic axis, however, have remained unclear.

We wished to test the idea that Sirt1 might mediate some of its effects on CR by controlling the somatotropic axis. Secretion of GH from the pituitary is directed by the hypothalamus, a region of the brain that is anatomically adjacent to the pituitary. We therefore used Cre/loxP technology to specifically ablate Sirtl in the brain, and examined the effects of this manipulation on somatotropic signaling in mice under normal and CR conditions. We found that these mutant mice are viable and have grossly normal brains, but show specific defects in the somatotropic axis. Moreover, Sirt 1 activity in the brain is required to mediate changes in somatotropic signaling and physical activity that occur in response to CR. This study establishes Sirt1 in the brain as the critical link between the GH and CR longevity pathways in mammals.

\section{Results and Discussion}

Generation of brain-specific Sirt1 knockout (BSKO) mice

We crossed a conditional allele of Sirt1 (Sirt $1^{\text {flox }}$ ) (Cheng et al. 2003) that had been backcrossed onto the C57Bl/6 genetic background (Chen et al. 2008) to a transgenic mouse strain in which Cre recombinase expression is driven by the Nestin promoter, also on the C57Bl/6 background (Tronche et al. 1999). Nestin-cre was always passed through the male germline. The nestin promoter drives Cre expression only in the cells that give rise to neurons, astrocytes, and glia. Sirt ${ }^{\text {flox/flox; }}$; NestinCre (BSKO) mice and Sirt1 $1^{\text {flox/flox }}$ littermate controls 
(referred to for simplicity as wild-type [WT]) were born at Mendelian ratios. BSKO mice were fertile and displayed grossly normal brain morphology (Fig. 1A). For all experiments, male mice were analyzed except where otherwise noted, but similar phenotypes were observed in both genders (data not shown).

The Sirt $1^{\text {flox }}$ allele has loxP sites flanking exon 4 of the Sirtl gene, which encodes the catalytic domain of the protein. Previous studies of these mice demonstrated that the excised version of this allele produces a stable (but nonfunctional) protein product that can be distinguished from wild-type Sirt1 protein by size (Cheng et al. 2003). To confirm excision of the Sirt $1^{\text {flox }}$ allele in BSKO mice, we analyzed brain homogenates from these animals and littermate controls by Western blotting. Sirt1 has two distinct protein isoforms in the brain (Fig. 1B, closed arrowheads). BSKO brains contained no wild-type Sirt1 protein, and only a small amount of the mutant protein (Fig. 1B, open arrowheads). A similar pattern was observed in lysates prepared from the hypothalamus of these animals (Supplemental Fig. S1). Importantly, in the pituitary, the majority of which arises from a nonneuronal lineage, we detected only wild-type Sirt1 in BSKO mice (Fig. 1C). Likewise, only wild-type Sirt1 protein was detected in peripheral tissues such as liver, muscle, and pancreas (Supplemental Fig. S1). Thus, NestinCre expression was confined to the neuronal lineage in our mice.

\section{BSKO mice are dwarfed and have reduced somatotropic signaling}

BSKO mice and their wild-type littermates were indistinguishable at birth and for their first month of life. However, beginning at 4-5 wk of age, we observed that BSKO mice were significantly smaller than their littermates, and that this size difference was maintained into adulthood
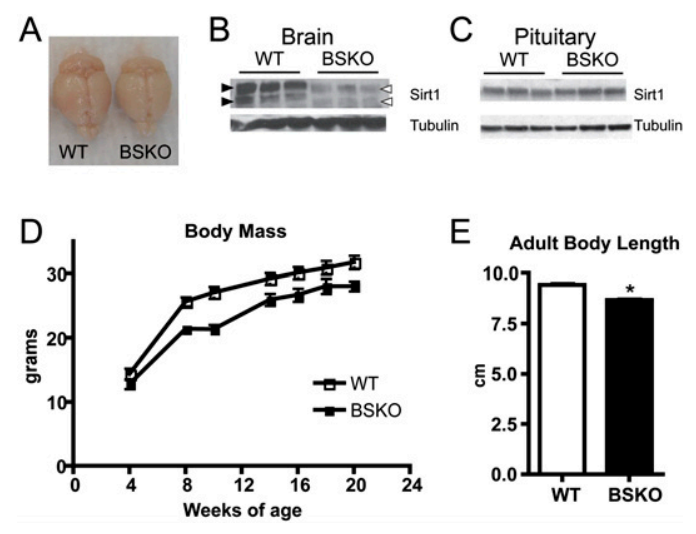

Figure 1. BSKO mice lack functional Sirt1 protein in the brain and are dwarfed. (A) Brains from wild-type (WT) and BSKO mice exhibit similar gross morphology. (B) Western blotting of Sirt1 protein in whole-brain lysates from wild-type and BSKO mice. Closed arrowheads indicate wild-type Sirt 1 isoforms, and open arrowheads indicate mutant isoforms. Tubulin is shown as a loading control. $(C)$ Western blotting of Sirt1 protein in pituitary lysates. $(D)$ Body mass of male wild-type and BSKO animals at the indicated ages; $n=6-10$ per genotype and time point. Curves are significantly different by repeated-measures ANOVA; $P<0.01$. (E) Snout-anus body length of male wild-type and BSKO animals at 10 wk of age; $n=6-10$ per genotype. All data are shown as mean \pm SEM. $\left(^{\star}\right) P<0.05$ by twotailed Student's $t$-test.
(Fig. 1D). This reduction in body mass was accompanied by a reduction in body length (Fig. 1E), and could not be attributed to a decrease in food consumption (Supplemental Fig. S1). Because somatotropic signaling is critical for post-weaning growth and for directing elongation of the long bones of the animal, we hypothesized that the dwarf phenotype of BSKO mice might arise from defects in the somatotropic axis.

To examine somatotropic signaling directly, we first measured serum GH levels in 4-wk-old mice, where GH levels are highest (Diaz-Torga et al. 2002). Mean circulating GH levels in BSKO mice were nearly 50\% lower than in wild-type littermate controls (Fig. 2A). We also measured IGF-1, which is released into the circulation predominantly by the liver in response to $\mathrm{GH}$ signaling. Serum IGF-1 levels, as well as IGF-1 mRNA levels in the liver, were significantly lower in adult BSKO as compared with wild-type animals (Fig. 2B,C). Finally, we examined transcript levels of genes that are expressed in a sexually dimorphic pattern in mouse liver in direct response to the different patterns of GH release in the two sexes (Waxman and O'Connor 2006). The expression pattern of these genes was significantly disrupted in BSKO mice of both sexes, providing additional evidence for alterations in GH secretion in adult BSKO mice (Supplemental Fig. S2).

Because GH is synthesized, stored, and released by the pituitary, we next examined this gland in BSKO mice. Pituitaries from BSKO animals were smaller than those of wild-type littermates, but displayed no major defects upon histological examination (Fig. 2D; Supplemental Fig. S3). The mass of the pituitaries of BSKO animals was reduced in comparison with wild-type littermates, even when the smaller body mass of BSKO mice was taken into account (Fig. 2E). Furthermore, each pituitary gland contained significantly less stored GH in BSKO mice as compared with controls, although the density of GHpositive cells was comparable between mice of both genotypes (Fig. 2F; Supplemental Fig. S3). To determine whether the defect in GH reflected a more general deficit in pituitary hormone production, we measured serum concentrations of three additional pituitary hormonesadrenocorticotropic hormone (ACTH), prolactin (PRL), and thyroid-stimulating hormone (TSH)-and two hormones produced by the thyroid in response to TSH (T3 and T4) (Fig. 2G). PRL concentrations were somewhat increased in BSKO mice as compared with wild type, while concentrations of ACTH, TSH, T3, and T4 were unchanged. Thus, the pituitary deficit of BSKO mice appears to be restricted to $\mathrm{GH}$.

Together, these findings imply that Sirt 1 acts in the brain to stimulate production and secretion of GH but not other pituitary hormones. Because development, proliferation, and function of the pituitary are under the control of the hypothalamus (for review, see Zhu et al. 2007), we hypothesize that the pituitary defects observed in BSKO mice are due to alterations in hypothalamic function in the absence of Sirt1. The production and release of $\mathrm{GH}$ is controlled by the opposing action of two hypothalamic neuropeptides: GH-releasing hormone and somatostatin (Low et al. 2001; Alba and Salvatori 2004). The expression of the mRNA encoding these neuropeptides is unaffected in BSKO mice (data not shown), and thus the mechanism by which hypothalamic Sirt1 acts to control the somatotropic axis is not certain. 


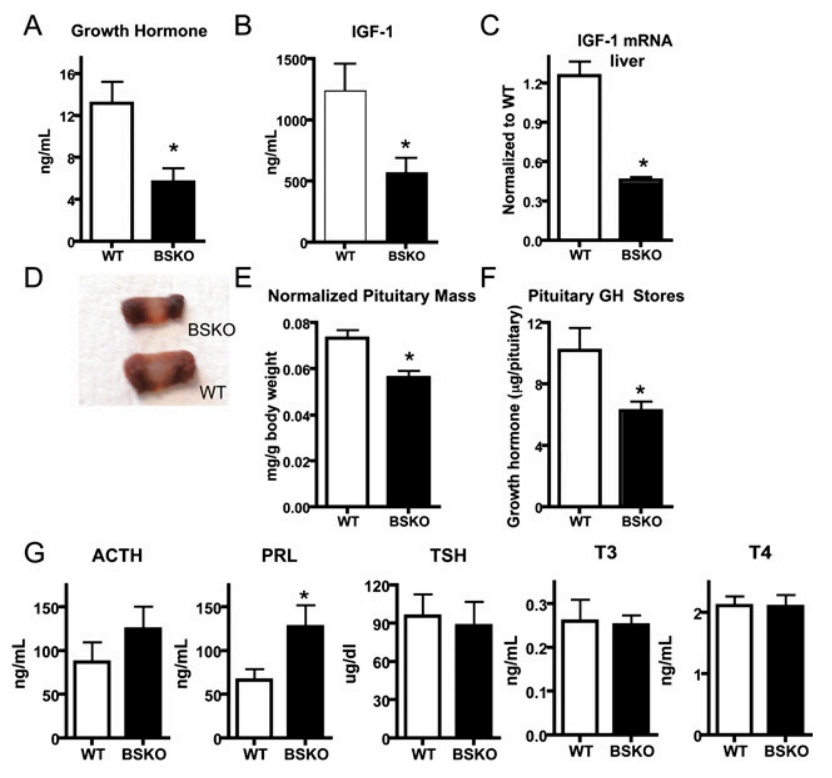

Figure 2. The somatotropic axis is disrupted in BSKO mice. $(A)$ Serum GH concentrations in 4-wk-old BSKO mice and littermate controls; $n=15-20$ per group. (B) Serum IGF-1 concentrations in 10wk-old BSKO mice and controls; $n=6-10$ per group. $(C)$ IGF-1 mRNA levels in BSKO liver normalized to wild-type (WT) levels; $n=$ 5 per group. $(D)$ Pituitaries from BSKO and wild-type littermates. $(E)$ Pituitary mass normalized to body mass of 10 -wk-old BSKO and wild-type mice; $n=6-10$ per group. (F) Pituitary GH stores in 10-wkold BSKO mice; $n=5-6$ per group. $(G)$ Measurements of additional pituitary hormones in BSKO and wild-type mice. For PRL, TSH, and $\mathrm{T} 4, n=10-14$ females per group. For T3 (females) and ACTH (males), $n=6-10$ per group. All data are shown as mean \pm SEM. $\left(^{\star}\right) P<0.05$ by two-tailed Student's $t$-test.

\section{Young BSKO mice display near-normal glucose metabolism}

Many mouse strains with decreased GH/IGF-1 signaling display improvements in baseline insulin sensitivity. To investigate whether this might also be true of BSKO mice, we measured fasted glucose and insulin in young adult BSKO animals and littermate controls. Young BSKO animals displayed a slight reduction in fasting blood glucose levels and a trend toward a reduction in fasted insulin levels that did not achieve statistical significance (Supplemental Fig. S4). We then assessed the performance of wild-type and BSKO mice in insulin and glucose tolerance tests (ITT and GTT, respectively). At 3 mo of age, BSKO and wild-type mice performed similarly on ITT (Fig. 3A). Since BSKO mice started with lower fasting glucose levels, the levels of blood glucose in these animals remained lower than that of wild type throughout the test (curves are significantly different by two-way ANOVA, $P<0.05$ ). Young wild-type and BSKO mice performed identically on the GTT (Fig. 3B). Thus, only minor differences in glucose tolerance and insulin sensitivity were detected in young BSKO mice.

We wondered whether these small differences in glucose metabolism could lead to protection against the metabolic changes brought on by a high-fat diet. To test this, we fed BSKO mice and littermate controls either a high-fat diet or matched control diet beginning at $6 \mathrm{wk}$ of age and continuing for $18 \mathrm{wk}$. All groups of mice ate a similar amount of food per gram of body weight /data not shown) and gained weight equally on the high-fat diet (Supplemental Fig. S5). By ITT, high-fat-fed BSKO mice appeared to remain somewhat more insulin-sensitive than wild-type mice fed the same diet, but similar protection was not observed by GTT (Supplemental Fig. S5). Thus, ablation of Sirt1 in the brain did not provide significant protection against the deleterious effects of a high-fat diet.

\section{Old BSKO mice display glucose intolerance and altered $C R$ responses}

Next, we asked whether BSKO animals would display the improvements in insulin sensitivity and glucose tolerance normally found in animals maintained on a CR diet. To test this, we placed BSKO mice and wild-type littermates on a CR diet, with CR mice being fed daily $60 \%$ by mass of the food consumed by ad libitum-fed controls. Animals of both genotypes lost weight similarly on CR (Supplemental Fig. S6). After 7 mo of CR, when all of the mice in this study were 10 mo old, we repeated the ITT
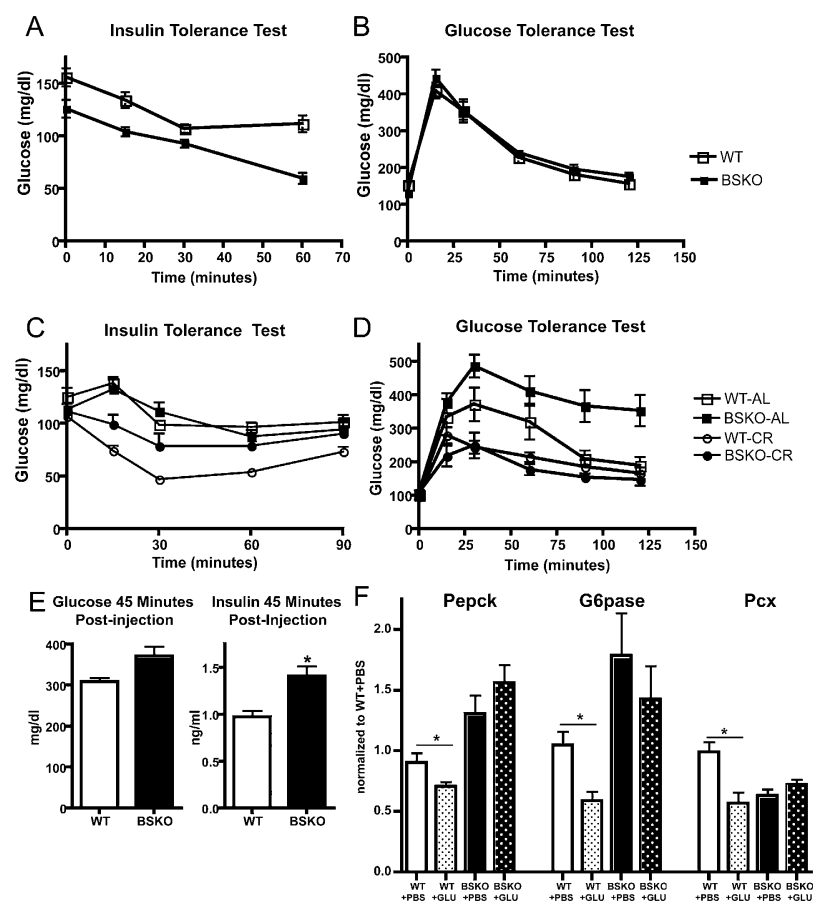

Figure 3. BSKO mice have altered glucose homeostasis with aging. (A) ITT on 3-mo-old BSKO and wild-type (WT) mice; $n=10-14$ per
group. Curves are significantly different by two-way ANOVA; $P<$ 0.05. (B) GTT on 3-mo-old BSKO and wild-type mice; $n=10-14$ per group. $(C)$ ITT on 10-mo-old mice of indicated diet and genotype groups; $n=6-8$ per group. (AL) Ad libitum fed; (CR) calorie restricted. WT-CR and BSKO-CR curves are significantly different by two-way ANOVA; $P<0.05$. (D) GTT on 10-mo-old mice of indicated diet and genotype groups; $n=6-8$ per group. All data are shown as mean \pm SEM. (E) Blood glucose and insulin levels in 1-yrold wild-type and BSKO mice $45 \mathrm{~min}$ after IP injection of $2 \mathrm{~g} / \mathrm{kg}$ glucose in PBS; $n=6-7$ per group. Insulin values are significantly different; $P<0.01$. The $P$-value for blood glucose comparison is 0.0727 by a two-tailed Student's $t$-test. (F) PEPCK, G6pase, and Pcx mRNA expression in liver of 0.5- to 1-yr-old female wild-type and BSKO mice $45 \mathrm{~min}$ following vehicle (PBS) or $2 \mathrm{~g} / \mathrm{kg}$ glucose (GLU) injection; $n=7-14$ per group. $\left(^{*}\right) P<0.05$ by two-tailed Student's $t$-test versus WT-PBS for each gene. 
and GTT experiments to determine the impact of CR on glucose homeostasis in BSKO animals. As in young animals, older ad libitum-fed wild-type and BSKO mice performed similarly on the ITT (Fig. 3C). As expected, CR dramatically increased the insulin sensitivity of wildtype animals. However, CR induced only a modest improvement in insulin sensitivity in BSKO animals, suggesting that neuronal Sirt1 is required to achieve the full benefit on insulin sensitivity that is normally conferred by CR. In contrast, the GTT revealed that BSKO and wildtype animals received a significant and equivalent benefit in glucose tolerance when maintained on a CR diet (Fig. 3D). This test also allowed us to compare older ad libitum animals to the young animals studied in Figure 3, A and B. Surprisingly, older BSKO animals developed severe glucose intolerance that was not observed in young mice.

We first hypothesized that the glucose intolerance of BSKO mice might arise from a failure of the pancreatic islets to produce sufficient insulin during the GTT. However, we found that BSKO mice of this age actually produce more insulin in response to glucose injection than wild type (Fig. 3E). We next reasoned that the glucose intolerance phenotype was unlikely to be due to a cell-autonomous effect on key peripheral tissues such as liver or muscle, since Sirt1 is ablated only in the neuronal lineages in BSKO animals (Supplemental Fig. S1). However, the ability of the liver to properly down-regulate gluconeogenesis in response to increasing insulin levels (as occur during a GTT) is in part under central control (for review, see Schwartz and Porte 2005). Insulinresponsive neurons in the hypothalamus direct the down-regulation of gluconeogenesis in the liver via the vagus nerve, which innervates the liver and other visceral organs. Mice in which the vagus nerve has been severed do not down-regulate gluconeogenic genes in response to insulin (Pocai et al. 2005). To determine if this brain-liver circuit was impaired in BSKO mice, we injected older adult (0.5- to 1-yr-old) wild-type and BSKO mice with a bolus of glucose, and after 45 min monitored expression of the mRNA encoding the gluconeogenic enzymes Pepck, G6pase, and Pcx as markers for gluconeogenesis in liver. In all cases, the genes were significantly downregulated in wild-type mice in response to glucose in- jection, whereas their expression was unchanged in BSKO mice (Fig. 3F). This finding supports the idea that the glucose intolerance of older BSKO mice arises from a breakdown in the brain-liver circuit that regulates glucose homeostasis.

The fact that CR rescues the glucose intolerance of older BSKO animals may be explained in two ways. First, it is possible that CR blocks the degeneration of the hypothalamic-liver circuit through a Sirt1-independent mechanism. Second, CR dramatically increases the insulin sensitivity of skeletal muscle, so it is possible that the muscles of BSKO mice on CR are able to take up the extra glucose present in the blood and thus mask the inability of the liver to halt gluconeogenesis. Breakdown of the brain-liver circuit may also help to explain our finding that BSKO mice apparently do not receive a full benefit in insulin sensitivity on CR, as measured by ITT; BSKO mice on CR may appear less insulin-sensitive because they are unable to turn off gluconeogenesis in response to an injection of insulin, and thus maintain higher blood glucose values throughout the test. It is also possible that Sirt1 plays some additional role in the central control of peripheral insulin sensitivity, since it is known from other genetic studies that the brain can, in fact, control insulin sensitivity through other pathways (Kahn et al. 2005).

\section{Neuronal Sirt1 regulates somatotropic signaling on $C R$}

A reduction in somatotropic signaling is one of the endocrine hallmarks of CR. Because our initial results demonstrated that neuronal Sirt1 regulates somatotropic signaling, we hypothesized that Sirtl activity in the brain might be required to direct down-regulation of this axis in response to CR. After wild-type and BSKO mice had been subjected to 10 wk of CR, we measured serum IGF-1 levels as a marker for somatotropic signaling in ad libitum and restricted animals of both genotypes. As expected, serum IGF-1 levels were significantly lower in wild-type mice on $\mathrm{CR}$ than in wild-type ad libitum controls. In contrast, IGF-1 levels remained unchanged in BSKO animals on CR as compared with ad libitum (Fig. 4A). Thus, Sirtl activity in the brain is absolutely
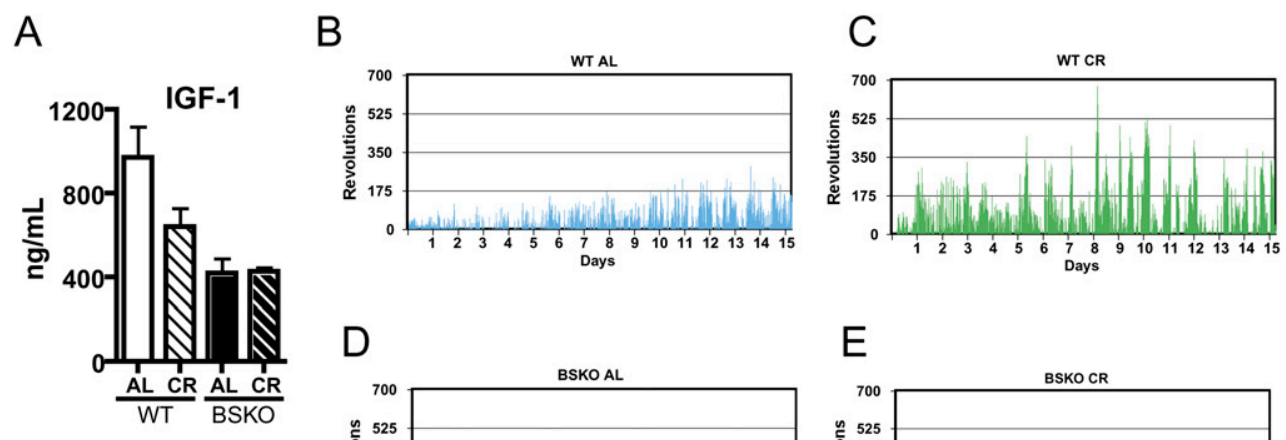

D
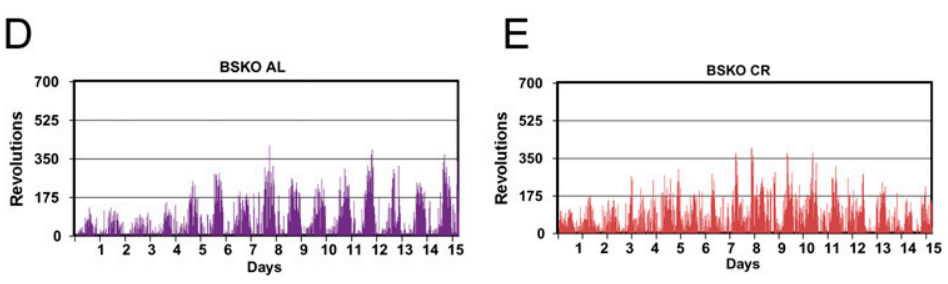

Figure 4. BSKO mice have altered endocrine and behavioral responses to CR. (A) Serum IGF-1 concentrations after 10 wk of $\mathrm{CR}$; $n=6-8$ per group. Interaction between diet and genotype is significant by two-way ANOVA; $P<0.05$. Data are shown as mean \pm SEM. $(B-E)$ Each bar represents the average number of revolutions run per 15 -min period over $15 \mathrm{~d}$ for mice of the indicated genotype and diet; $n=5$ per group. 
required to regulate the somatotropic axis in response to diet.

Since Sirt1 appears to be a positive regulator of $\mathrm{GH}$ production and signaling, our findings also suggest that Sirtl activity in the hypothalamus might decrease during CR. Because no changes in Sirtl protein levels were detected in the hypothalamus or pituitary in mice fed a CR diet (Supplemental Fig. S7), it is likely that other factors such as changes in the NAD/NADH ratio act to decrease Sirtl activity in the hypothalamus during CR. This is in contrast to other tissues, such as skeletal muscle and white adipose tissue, where Sirtl activity has been reported to increase in response to CR. However, previous findings indicate that Sirt1 activity in the murine liver decreases during CR (Chen et al. 2008). Thus, the liver and hypothalamus may be members of a subset of tissues in which it is a decrease, rather than an increase, in Sirtl function that mediates physiological changes during CR.

\section{Neuronal Sirt1 regulates physical activity on ad libitum and $C R$ diets}

A well-established behavioral consequence of CR in mice is up-regulation of physical activity, presumably a correlate of increased foraging behavior. Previous work using animals lacking Sirt1 throughout the body demonstrated that Sirt 1 was required for this up-regulation of activity in response to CR (Chen et al. 2005). However, this study left open the question of whether Sirt 1 activity is required in the nervous system or the periphery to generate this behavioral change. To test the requirement for Sirtl in the brain in the behavioral response to $C R$, we used running wheels to monitor physical activity in ad libitum and restricted animals of both genotypes for a 2-wk period beginning after $7 \mathrm{mo}$ of CR. As has been shown previously, wild-type animals dramatically up-regulated their activity in response to CR (Fig. 4B,C). BSKO animals were somewhat more active than wild-type animals on an ad libitum diet (Fig. 4D), and their activity did not increase-and, in fact, slightly decreased-on the CR diet (Fig. 4E). We conclude that Sirtl activity is required specifically in the brain to regulate physical activity in ad libitum mice and to drive the changes in activity brought on by CR.

\section{Conclusions}

This study brings together three major pathways known to affect aging - CR, the somatropic axis, and sirtuins-in what is likely a single mechanism operating in the brain. Our findings suggest that CR triggers a reduction in Sirt 1 activity in hypothalamic neurons governing somatotropic signaling to lower this axis, in contrast with the activation of Sirt1 by CR in many other tissues. Sirt1 may have evolved to positively regulate the somatotropic axis, as it does insulin production in $\beta$ cells, to control mammalian health span and life span in an overarching way. However, the fact that Sirtl is a positive regulator of the somatotropic axis may complicate attempts to increase murine life span by whole-body activation of this sirtuin.

It is interesting that Sirt 1 activity in the brain governs the production of $\mathrm{GH}$, but not other pituitary hormones, even though the production of all pituitary hormones is regulated at least in part by signals from the hypothalamus. It will be of interest to dissect the function of this sirtuin in specific brain regions with more targeted knockout strategies. Likewise, it will be important to study Sirt 1 in the pituitary itself to address whether this sirtuin also controls the other major endocrine axes.

\section{Materials and methods}

\section{Animals}

All animal procedures were carried out in accordance with the Massachusetts Institute of Technology Committee on Animal Care. Mice were group-housed in a 12-h light/12-h dark cycle at controlled temperature $\left(25^{\circ} \mathrm{C} \pm 1{ }^{\circ} \mathrm{C}\right)$. For high-fat diet studies, mice were fed diets with $60 \%$ or $10 \%$ of calories from fat (high fat and control, respectively; Research Diets, Inc.). For CR studies, the amount of food consumed by ad libitum control mice was determined weekly, and CR mice were fed $60 \%$ of that value daily. For GTT and ITT, mice were fasted overnight prior to injection with either $2 \mathrm{~g} / \mathrm{kg}$ glucose (Sigma) or $1 \mathrm{IU} / \mathrm{kg}$ porcine insulin (Sigma) delivered in sterile saline. Blood glucose was measured at the indicated time points using a LifeScan OneTouch glucometer. Activity was measured by individually housing animals with low-profile wireless running wheels (Med Associates, Inc.). For tests of the response of animals to a bolus of glucose, mice were fasted for $14 \mathrm{~h}$ and then injected with either $2 \mathrm{~g} / \mathrm{kg}$ glucose or an equivalent volume of saline vehicle and were analyzed 45 min after injection.

\section{Western blotting}

Tissues were lysed in TNT buffer (20 mM Tris at pH 8.0, $150 \mathrm{mM} \mathrm{NaCl}$, $1 \%$ Triton X-100) supplemented with protease inhibitors (Roche), and blotted with antibodies against Sirt1 (Millipore) and tubulin (Sigma).

\section{Hormone measurements}

Serum for hormone measurements was collected by retro-orbital puncture under isofluorane anesthesia. ELISA kits for measuring hormone concentrations (listed in the Supplemental Material) were used according to the manufacturer's instructions.

\section{Quantitative RT-PCR}

Total RNA was isolated using Trizol reagent (Invitrogen) and was treated with DNase (Promega) prior to reverse transcription with MMLV reverse transcriptase (Invitrogen) and random hexamer primers (New England Biolabs). Real-time PCR reactions were performed on a LightCycler $480 \mathrm{II}$ (Roche) using iQ SYBR Green Supermix (Bio-Rad). The relative abundance of transcripts of interest was calculated by first normalizing to a housekeeping gene ( $\beta$-actin or 18S RNA) and then normalizing to wild-type controls using the $2^{-\Delta \Delta \mathrm{CT}}$ method. Primer sequences were taken from the Harvard Primer Bank (Wang and Seed 2003; Spandidos et al. 2008). For sexspecific gene expression in the liver, primer sequences were as described (Holloway et al. 2008).

\section{Acknowledgments}

We thank Eric Bell and Dudley Lamming for helpful discussions and comments on the manuscript. D.E.C. and A.M.S. were supported by funding from CHDI, Inc., and the Hereditary Disease Foundation. G.D. was supported by a fellowship from the American Parkinson's Disease Association. L.G. is supported by the NIH and by the Paul F. Glenn Foundation.

\section{References}

Alba M, Salvatori R. 2004. A mouse with targeted ablation of the growth hormone-releasing hormone gene: A new model of isolated growth hormone deficiency. Endocrinology 145: 4134-4143.

Al-Regaiey KA, Masternak MM, Bonkowski M, Sun L, Bartke A. 2005. Long-lived growth hormone receptor knockout mice: Interaction of reduced insulin-like growth factor $\mathrm{i}$ /insulin signaling and caloric restriction. Endocrinology 146: 851-860. 
Banks AS, Kon N, Knight C, Matsumoto M, Gutierrez-Juarez R, Rossetti L, Gu W, Accili D. 2008. SirT1 gain of function increases energy efficiency and prevents diabetes in mice. Cell Metab 8: 333-341.

Barger JL, Kayo T, Pugh TD, Prolla TA, Weindruch R. 2008a. Short-term consumption of a resveratrol-containing nutraceutical mixture mimics gene expression of long-term caloric restriction in mouse heart. Exp Gerontol 43: 859-866.

Barger JL, Kayo T, Vann JM, Arias EB, Wang J, Hacker TA, Wang Y, Raederstorff D, Morrow JD, Leeuwenburgh C, et al. 2008b. A low dose of dietary resveratrol partially mimics caloric restriction and retards aging parameters in mice. PLoS One 3: e2264. doi: 10.1371/journal. pone.0002264.

Boily G, Seifert EL, Bevilacqua L, He XH, Sabourin G, Estey C, Moffat C, Crawford S, Saliba S, Jardine K, et al. 2008. SirT1 regulates energy metabolism and response to caloric restriction in mice. PLOS ONE 3: e1759. doi: 10.1371/journal.pone.0001759.

Bonkowski MS, Rocha JS, Masternak MM, Al Regaiey KA, Bartke A. 2006. Targeted disruption of growth hormone receptor interferes with the beneficial actions of calorie restriction. Proc Natl Acad Sci 103: 7901-7905.

Bordone L, Guarente L. 2005. Calorie restriction, SIRT1 and metabolism: Understanding longevity. Nat Rev Mol Cell Biol 6: 298-305.

Bordone L, Cohen D, Robinson A, Motta MC, van Veen E, Czopik A, Steele AD, Crowe H, Marmor S, Luo J, et al. 2007. SIRT1 transgenic mice show phenotypes resembling calorie restriction. Aging Cell 6: 759-767.

Chen D, Steele AD, Lindquist S, Guarente L. 2005. Increase in activity during calorie restriction requires Sirt1. Science 310: 1641. doi: 10.1126/science.1118357.

Chen D, Bruno J, Easlon E, Lin SJ, Cheng HL, Alt FW, Guarente L. 2008. Tissue-specific regulation of SIRT1 by calorie restriction. Genes \& Dev 22: 1753-1757.

Cheng HL, Mostoslavsky R, Saito S, Manis JP, Gu Y, Patel P, Bronson R, Appella E, Alt FW, Chua KF. 2003. Developmental defects and p53 hyperacetylation in Sir2 homolog (SIRT1)-deficient mice. Proc Natl Acad Sci 100: 10794-10799.

Cohen HY, Miller C, Bitterman KJ, Wall NR, Hekking B, Kessler B, Howitz KT, Gorospe M, de Cabo R, Sinclair DA. 2004. Calorie restriction promotes mammalian cell survival by inducing the SIRT1 deacetylase. Science 305: 390-392.

Diaz-Torga G, Feierstein C, Libertun C, Gelman D, Kelly MA, Low MJ, Rubinstein M, Becu-Villalobos D. 2002. Disruption of the D2 dopamine receptor alters GH and IGF-I secretion and causes dwarfism in male mice. Endocrinology 143: 1270-1279.

Guarente L. 2009. Cell biology. Hypoxic hookup. Science 324: 12811282.

Holloway MG, Miles GD, Dombkowski AA, Waxman DJ. 2008. Liverspecific hepatocyte nuclear factor- $4 \alpha$ deficiency: Greater impact on gene expression in male than in female mouse liver. Mol Endocrinol 22: $1274-1286$.

Holzenberger M, Dupont J, Ducos B, Leneuve P, Geloen A, Even PC, Cervera P, Le Bouc Y. 2003. IGF-1 receptor regulates lifespan and resistance to oxidative stress in mice. Nature 421: 182-187.

Imai S, Johnson FB, Marciniak RA, McVey M, Park PU, Guarente L. 2000. Sir2: An NAD-dependent histone deacetylase that connects chromatin silencing, metabolism, and aging. Cold Spring Harb Symp Quant Biol 65: 297-302.

Kahn BB, Alquier T, Carling D, Hardie DG. 2005. AMP-activated protein kinase: Ancient energy gauge provides clues to modern understanding of metabolism. Cell Metab 1: 15-25.

Ladiges W, Van Remmen H, Strong R, Ikeno Y, Treuting P, Rabinovitch P, Richardson A. 2009. Lifespan extension in genetically modified mice. Aging Cell 8: 346-352.

Li Y, Xu W, McBurney MW, Longo VD. 2008. SirT1 inhibition reduces IGF-I/IRS-2/Ras/ERK1/2 signaling and protects neurons. Cell Metab 8: $38-48$.

Lin SJ, Defossez PA, Guarente L. 2000. Requirement of NAD and SIR2 for life-span extension by calorie restriction in Saccharomyces cerevisiae. Science 289: 2126-2128.

Low MJ, Otero-Corchon V, Parlow AF, Ramirez JL, Kumar U, Patel YC, Rubinstein M. 2001. Somatostatin is required for masculinization of growth hormone-regulated hepatic gene expression but not of somatic growth. J Clin Invest 107: 1571-1580.
Pearson KJ, Baur JA, Lewis KN, Peshkin L, Price NL, Labinskyy N, Swindell WR, Kamara D, Minor RK, Perez E, et al. 2008. Resveratrol delays age-related deterioration and mimics transcriptional aspects of dietary restriction without extending life span. Cell Metab 8: 157168.

Pfluger PT, Herranz D, Velasco-Miguel S, Serrano M, Tschop MH. 2008. Sirtl protects against high-fat diet-induced metabolic damage. Proc Natl Acad Sci 105: 9793-9798.

Pocai A, Lam TK, Gutierrez-Juarez R, Obici S, Schwartz GJ, Bryan J, Aguilar-Bryan L, Rossetti L. 2005. Hypothalamic K(ATP) channels control hepatic glucose production. Nature 434: 1026-1031.

Rogina B, Helfand SL. 2004. Sir2 mediates longevity in the fly through a pathway related to calorie restriction. Proc Natl Acad Sci 101: 15998-16003.

Schwartz MW, Porte D Jr. 2005. Diabetes, obesity, and the brain. Science 307: 375-379.

Spandidos A, Wang X, Wang H, Dragnev S, Thurber T, Seed B. 2008. A comprehensive collection of experimentally validated primers for Polymerase Chain Reaction quantitation of murine transcript abundance. BMC Genomics 9: 633. doi: 10.1186/1471-2164-9-633.

Tatar M, Bartke A, Antebi A. 2003. The endocrine regulation of aging by insulin-like signals. Science 299: 1346-1351.

Tronche F, Kellendonk C, Kretz O, Gass P, Anlag K, Orban PC, Bock R, Klein R, Schutz G. 1999. Disruption of the glucocorticoid receptor gene in the nervous system results in reduced anxiety. Nat Genet 23: 99-103.

Wang X, Seed B. 2003. A PCR primer bank for quantitative gene expression analysis. Nucleic Acids Res 31: e154. doi: 10.1093/nar/ gng154.

Waxman DJ, O'Connor C. 2006. Growth hormone regulation of sexdependent liver gene expression. Mol Endocrinol 20: 2613-2629.

Zhu X, Gleiberman AS, Rosenfeld MG. 2007. Molecular physiology of pituitary development: Signaling and transcriptional networks. Physiol Rev 87: 933-963. 


\section{Erratum}

Genes \& Development 23: 2812-2817 (2009)

\section{Neuronal SIRT1 regulates endocrine and behavioral responses to calorie restriction}

Dena E. Cohen, Andrea M. Supinski, Michael S. Bonkowski, Gizem Donmez, and Leonard P. Guarente

Due to an error during figure preparation for the above-mentioned paper, one of the graphs in Figure 4 was inadvertently duplicated such that the graphs in panels B and E appeared identical. A corrected version of the figure is shown below, such that B remains as originally presented and E is replaced by the correct, nonduplicated graph. This error in no way affects the conclusions of the paper.

The authors apologize for any confusion caused by this error.

Please note that this has been corrected in the online version of this paper.

A

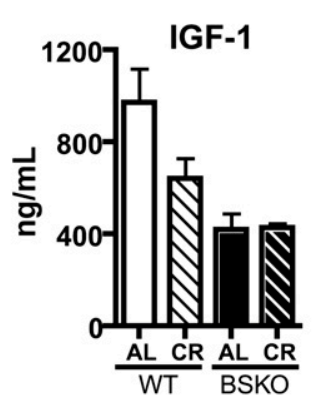

B

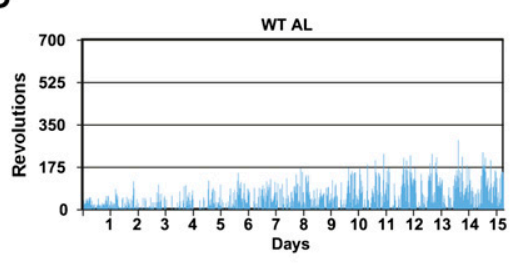

D

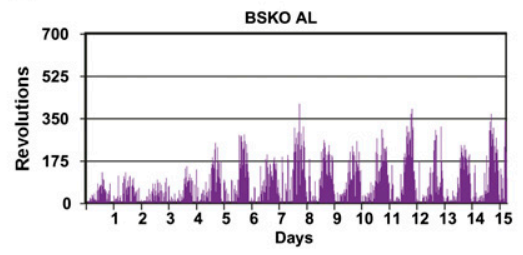

C

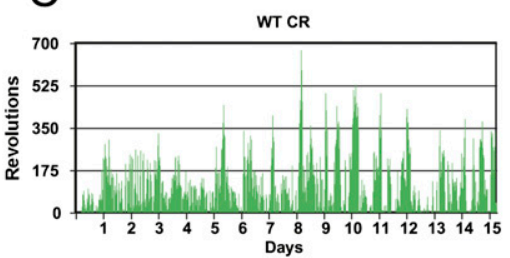

$\mathrm{E}$

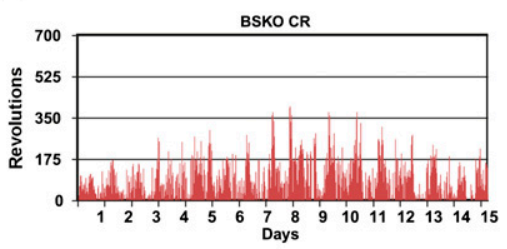




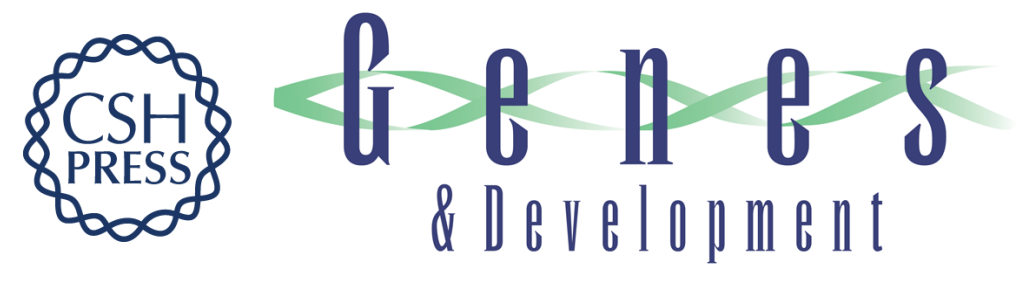

\section{Neuronal SIRT1 regulates endocrine and behavioral responses to calorie restriction}

Dena E. Cohen, Andrea M. Supinski, Michael S. Bonkowski, et al.

Genes Dev. 2009, 23:

Access the most recent version at doi:10.1101/gad.1839209

\section{Supplemental http://genesdev.cshlp.org/content/suppl/2009/11/30/23.24.2812.DC1 \\ Material}

Related Content Erratum: Neuronal SIRT1 regulates endocrine and behavioral responses to calorie restriction

Dena E. Cohen, Andrea M. Supinski, Michael S. Bonkowski, et al.

Genes Dev. March , 2010 24: 516

References This article cites 34 articles, 12 of which can be accessed free at:

http://genesdev.cshlp.org/content/23/24/2812.full.html\#ref-list-1

Articles cited in:

http://genesdev.cshlp.org/content/23/24/2812.full.html\#related-urls

License

Email Alerting

Service

Receive free email alerts when new articles cite this article - sign up in the box at the top right corner of the article or click here.

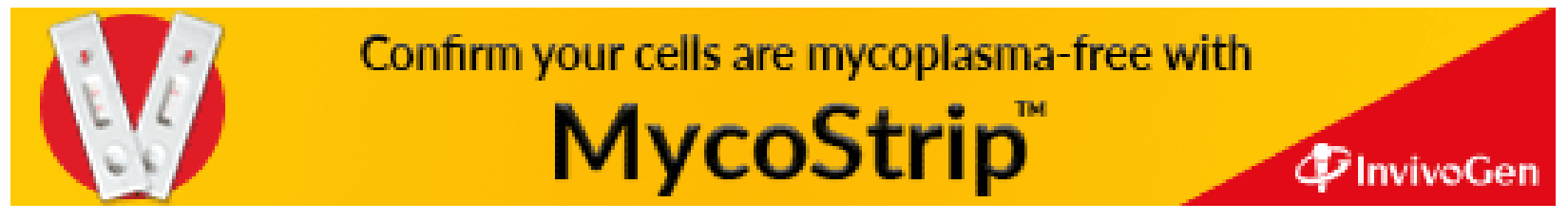

